\title{
Oxidation of $N, N$-Disubstituted Hydroxylamines to Nitrones: The Search for More Sustainable Selective and Practical Stoichiometric Oxidants
}

\author{
Camilla Matassini ${ }^{\star a b}$ and Francesca Cardona ${ }^{\star a b}$
}

\begin{abstract}
Nitrones are key intermediates for the total synthesis of nitrogen-containing natural products and analogues. The direct oxidation of the corresponding $\mathrm{N}, \mathrm{N}$-disubstituted hydroxylamines is one of the most straightforward methods to access such compounds. In this account, we describe the state of the art of this oxidative transformation using stoichiometric reagents. We will focus on the efforts made in the last 25 years to oxidize polyhydroxylated cyclic hydroxylamines, due to our long-standing interest in the total synthesis of alkaloids and other glycomimetics. Particular attention will be devoted to the discussion of the regioselectivity issue arising when unsymmetrical and highly functionalized substrates are involved.
\end{abstract}

Keywords: $N, N$-Disubstituted hydroxylamines $\cdot$ Nitrones $\cdot$ Oxidation $\cdot$ Regioselectivity $\cdot$ Stoichiometric reagents

\section{Introduction}

Nitrones are extremely valuable compounds used as spin traps in biological studies and as drugs for age-related diseases. ${ }^{[1]}$ Moreover, they are useful building blocks for the total synthesis of nitrogencontaining natural products and their biologically relevant analogues. Their versatility as synthetic intermediates is due to the ability of nitrones to react as electrophiles with organometallic reagents, ${ }^{[2]}$ or as 1,3-dipoles in [3+2] cycloaddition reactions. ${ }^{[3]}$ In addition, they are suitable substrates in $[3+3]$ cycloadditions, or reagents in new metal-mediated reactions. ${ }^{[4,5]}$

The most common procedures employed for the synthesis of nitrones $\mathbf{1}$ are reported in Scheme 1 and can be divided into two main strategies: i) oxidation of $\mathrm{N}, \mathrm{N}$-disubstituted hydroxylamines $\mathbf{2}$ or

${ }^{*}$ Correspondence: Prof. F. Cardona, Dr. C. Matassini E-mail: francesca.cardona@unifi.it,

camilla.matassini@unifi.it

aDipartimento di Chimica, University of Firenze,

Via della Lastruccia 3-13

50019 Sesto Fiorentino (FI), Italy

${ }^{\mathrm{b}}$ Associated with CNR-INO

Via N. Carrara 1, Sesto Fiorentino (FI), Italy secondary amines $\mathbf{3}^{[6]}$ and ii) condensation of aldehydes $\mathbf{4}$ with $N$-monosubstituted hydroxylamines 5. ${ }^{[2,7]}$ An alternative strategy developed in our group is the direct oxidation of imines $\mathbf{6}$ to nitrones $1{ }^{[8]}$ Moreover, an iridium-catalyzed reductive approach to nitrones from $N$-hydroxyamides $\mathbf{7}$ was recently reported. ${ }^{[9]}$ As highlighted in Scheme 1, this review is focused on the oxidation of hydroxylamines $\mathbf{2}$ to nitrones $\mathbf{1}$.

Actually, $N, N$-disubstituted hydroxylamines 2 can be oxidized under much milder conditions than amines $\mathbf{3}$ (often occurring as intermediates in amine oxidation), affording nitrones $\mathbf{1}$ in excellent yields. Hydroxylamine oxidation often requires the same or similar reagents able to convert alcohols to the corresponding aldehydes or ketones. However, while alcohol oxidation is widely investigated by researchers belonging to different areas, ${ }^{[10,11]}$ the corresponding oxidation of hydroxylamines is much less studied and the aim of this review is to resume and collate the efforts of the researchers to find more sustainable, practical and selective stoichiometric oxidants for this transformation.

In particular, in this account we will focus on the oxidation of cyclic enantiopure hydroxylamines since the corresponding cyclic nitrones are common and useful precursors for the synthesis of natural alkaloids. ${ }^{[3 c]}$ Moreover, while the preparation of hydroxylamines is not trivial in the case of acyclic compounds, cyclic compounds can be readily obtained through a double nucleophilic displacement with hydroxylamine. ${ }^{[2]}$

We will limit the discussion to stoi- chiometric oxidants and we will discuss the advantages and disadvantages of the different procedures, also, when possible, by comparing yields and selectivity on the same substrates.

\section{Stoichiometric Oxidants for the Conversion of $\mathrm{N}, \mathrm{N}$-Disubstituted Hydroxylamines 2 to Nitrones 1 (from 1993 to 2017)}

Early stoichiometric oxidants for the synthesis of acyclic and cyclic nitrones from $N, N$-disubstituted hydroxylamines were already reviewed, ${ }^{[12]}$ and include the use of oxygen or air (even if for very long reaction times), potassium ferricyanide, potassium permanganate, $\mathrm{N}$-bromosuccinimide, iodine, ceric ammonium nitrate, $t$-butylhydroperoxide, mer-

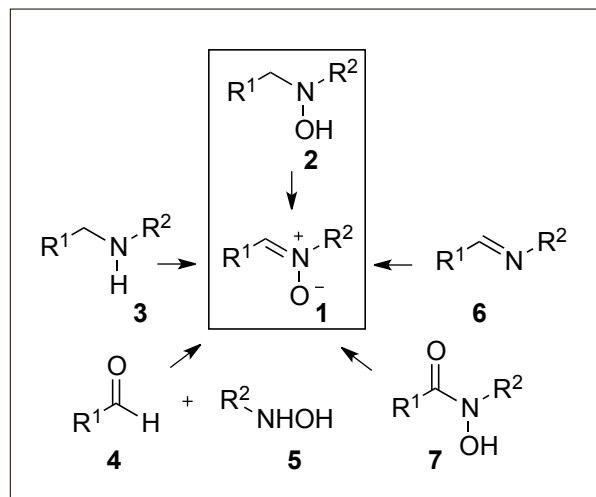

Scheme 1. Eligible precursors for the synthesis of nitrones 1 . 
curic oxide, diethyl azodicarboxylate and hydrogen peroxide. ${ }^{[12,13]}$

Brandi and co-workers reported in 1993 an efficient strategy for the synthesis of cyclic nitrones 9 starting from L-tartaric acid and using 2 equiv. $\mathrm{HgO}$ (yellow) in $\mathrm{CH}_{2} \mathrm{Cl}_{2}$ for the final oxidation step of hydroxylamines 8 (Scheme 2 ) ${ }^{[14]}$ In this case, the $C_{2}$ symmetry of hydroxypyrrolidine $\mathbf{8}$ assured the obtainment of enantiopure nitrones 9.

However, it has to be taken into account that when unsymmetrical hydroxylamines are employed, there is a regioselectivity issue: two regioisomeric nitrones can be formed with the selectivity depending on each individual case. This is clearly evidenced in Scheme 3, showing the oxidation of hydroxylamine $\mathbf{1 0}$, which gave nitrones $\mathbf{1 1}$ and $\mathbf{1 2}$ in 9:1 ratio. ${ }^{[15]}$

The observed selectivity was rational-

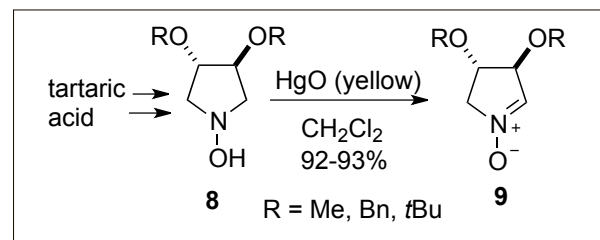

Scheme 2. Oxidation of cyclic hydroxylamines 8 with $\mathrm{HgO}$ (yellow).

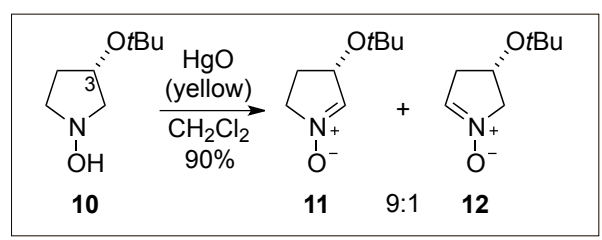

Scheme 3: Oxidation of unsymmetrically substituted hydroxylamine $\mathbf{1 0}$ with $\mathrm{HgO}$ (yellow).

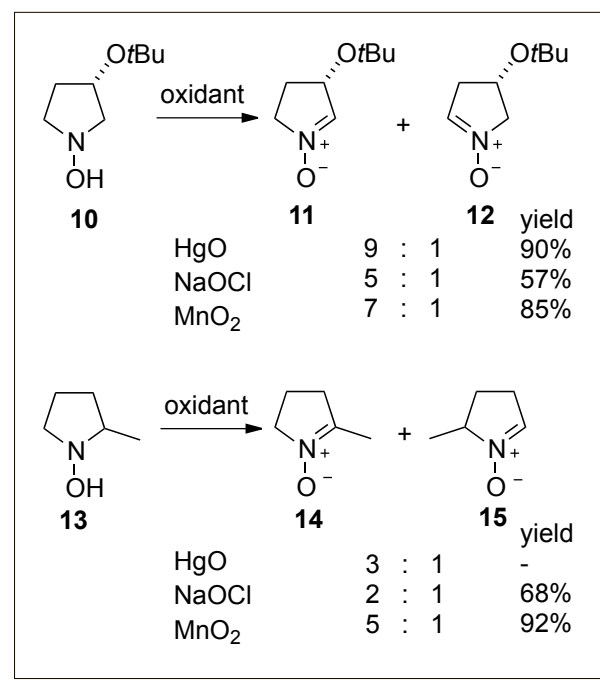

Scheme 4. Oxidation of unsymmetrically substituted hydroxylamines 10 and 13 with $\mathrm{HgO}, \mathrm{NaOCl}$ and $\mathrm{MnO}_{2}$. ized by studying the oxidation of a deuterated hydroxylamine, and arises from a kinetic preference for the cleavage of the $\mathrm{C}-\mathrm{H}$ bond anti to the tert-butoxy group at $\mathrm{C}(3)$ of the hydroxypyrrolidine, thus giving isomer $\mathbf{1 1}$ as the favored one.[16] This behavior is related to the electronegative nature of the substituent $(\mathrm{O} t \mathrm{Bu}$, in this case), with nearly complete regioselectivity reached with analogue of $\mathbf{1 0}$ bearing the OCOPh substituent at $\mathrm{C}(3)$, and lower selectivity encountered with less electronwithdrawing groups. ${ }^{[15,17]}$

Use of mercury salts in excess amounts, although being universal, efficient and successful in most cases, ${ }^{[3 a, 7,18]}$ should be avoided for safety reasons due to the high toxicity of these oxidants.

Goti and co-workers in 1999 observed that sodium hypochlorite $(\mathrm{NaOCl})$ was able to promote the oxidation of hydroxylamines to nitrones. ${ }^{[19]}$ This inexpensive, safe, benign oxidant (the only byproduct being sodium chloride) allowed to oxidize a panel of ten hydroxylamines including acyclic and cyclic ones in moderate to good yields (50-92\%). An aqueous solution (5 or $13 \%$ ) of $\mathrm{NaOCl}$ (ca. 1.3 equiv.) was added to a $0.5 \mathrm{M}$ solution of hydroxylamine in $\mathrm{CH}_{2} \mathrm{Cl}_{2}$ at $0{ }^{\circ} \mathrm{C}$, and reacted at room temperature for $2-25 \mathrm{~h}$. The procedure is very simple, requiring only a separation of the biphasic mixture before purification of the product. The reaction rate for the oxidation dramatically increased when the reactions were performed at $\mathrm{pH}=2.8$; however, lower selectivity and yields were afforded at acidic $\mathrm{pH}$. The only limitation of this method appeared to be the low regioselectivity in the oxidation of 3-substituted hydroxypyrrolidines (Scheme 4).

The introduction in 2001 of $\mathrm{MnO}_{2}$ as the oxidant, used in small excess (1.5 equiv.), represented one of the major breakthrough in the field. ${ }^{[20]}$ This reagent was used for the oxidation of several structurally different hydroxylamines, and good results in terms of yields (85-96\%) and regioselectivity were obtained. For instance, the oxidation of hydroxylamine 10 with $\mathrm{MnO}_{2}$ afforded a mixture of the two regioisomeric nitrones $\mathbf{1 1}$ and $\mathbf{1 2}$ in 7:1 ratio and $85 \%$ yield (Scheme 4), showing only a lower selectivity with respect to $\mathrm{HgO}^{[15]}(9: 1)$, but a better one with respect to bleach ${ }^{[19]}(5: 1)$.

In the case of $\alpha$-substituted hydroxylamines such as $\mathbf{1 3}$ (Scheme 4), the ketonitrone 14 and the aldonitrone 15 originate by dehydrogenation of the more substituted or the less substituted $\alpha$-carbon, respectively, with the first one being favored by thermodynamic effects and the latter by statistical effects. This regioselectivity issue is still an unmet goal and may depend on both the steric hindrance of the peculiar hydroxylamine and oxidant employed in each case. ${ }^{[21]}$ The oxidation of hydroxylamine 13 with $\mathrm{HgO}, \mathrm{NaOCl}$ and $\mathrm{MnO}_{2}$ is shown in Scheme 4. In all cases the ketonitrone 14 was the major product; however, the selectivity was higher with $\mathrm{MnO}_{2}$ as the oxidant, compared to $\mathrm{HgO}^{[22]}$ (5:1 vs $3: 1$ ), while bleach ${ }^{[19]}$ gave the lowest selectivity (2:1).

The scope of $\mathrm{MnO}_{2}$ was extended in 2002 to the oxidation of several $\mathrm{N}$-alkyl$N$-glycosyl hydroxylamines, precursors of enantiomerically pure $N$-glycosyl nitrones. ${ }^{[23]}$ These reactions generally occur with complete regioselectivity in favor of the aldonitrone, as shown in Scheme 5 for the selective oxidation of $\mathbf{1 6}$ to nitrone $\mathbf{1 7}$. The oxidation of 18 with $\mathrm{MnO}_{2}$ led to a complex mixture of compounds, probably due to the instability of $C$-unsubstituted nitrone 19. However, when $\mathbf{1 8}$ was treated with DDQ (2,3-dichloro-5,6-dicyano-1,4benzoquinone), the aldonitrone 19 was regioselectively formed and immediately reacted with dimethyl maleate, affording a 1:1 mixture of two isoxazolidine adducts 20 and 21. A previous example of $N, N$ disubstituted hydroxylamine oxidation was reported using unsubstituted $p$-benzoquinone by Ali and co-workers. ${ }^{[24]}$

An old stoichiometric oxidation with lead dioxide in dichloromethane was reported allowing the successful preparation

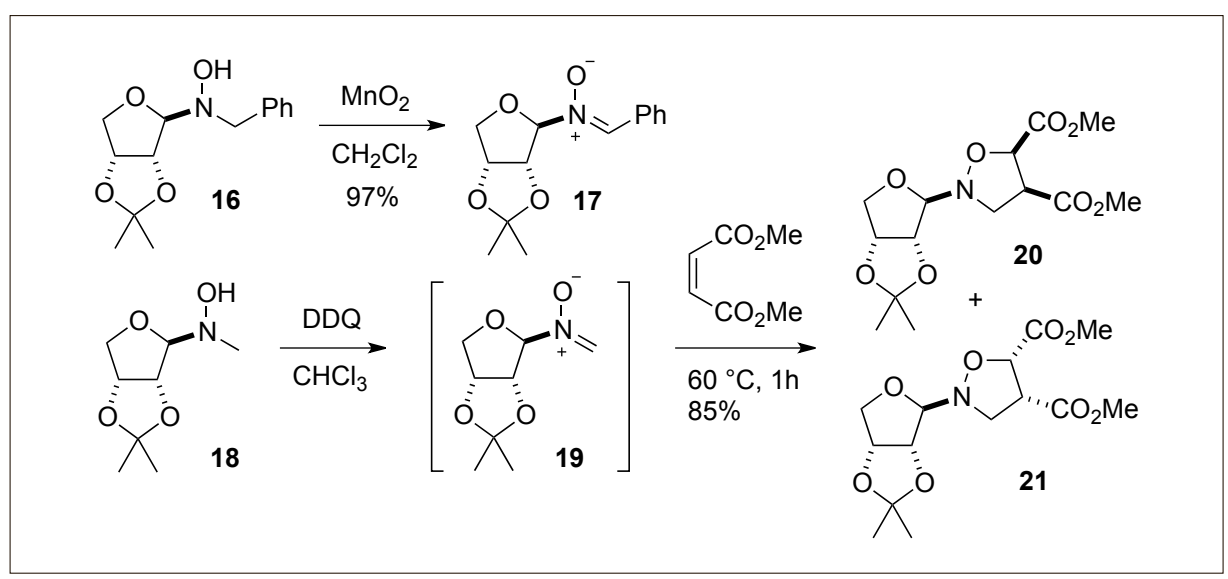

Scheme 5. Oxidation of $\mathrm{N}$-glycosyl hydroxylamines 16 and 18 with $\mathrm{MnO}_{2}$ and DDQ. 


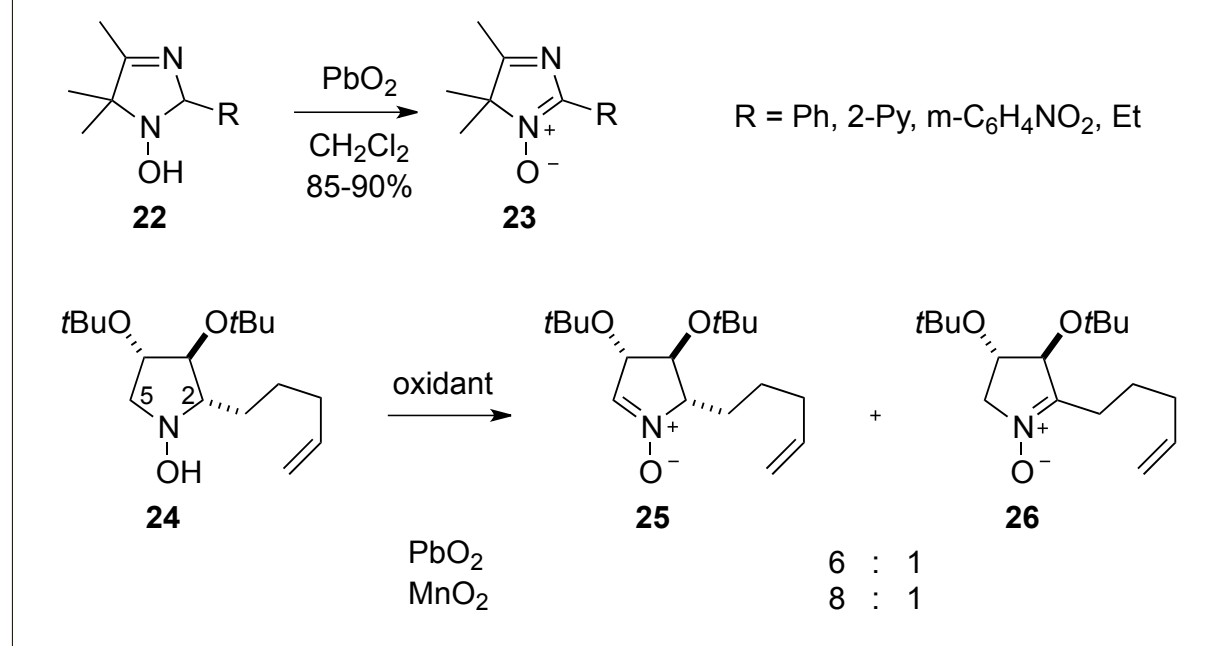

Scheme 6. Oxidation of $\mathrm{N}, \mathrm{N}$-disubstituted hydroxylamines with $\mathrm{PbO}_{2}$.

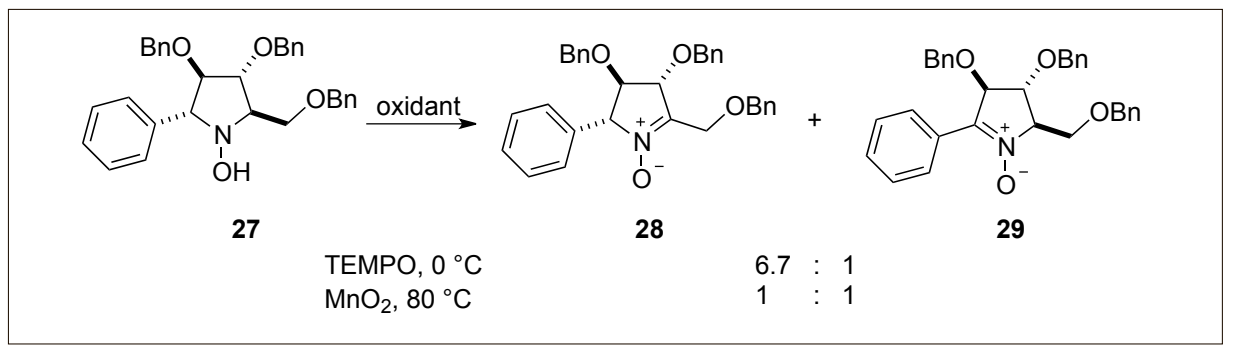

Scheme 7. Oxidation of hydroxylamine 27.

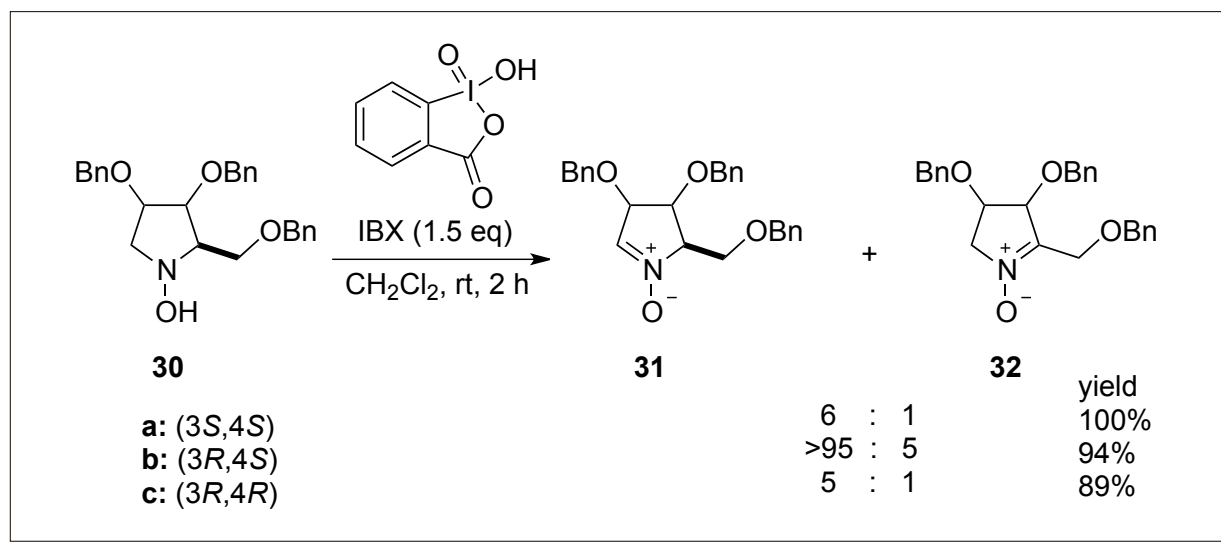

Scheme 8. Oxidation with IBX of carbohydrate-derived hydroxylamines $\mathbf{3 0}$.

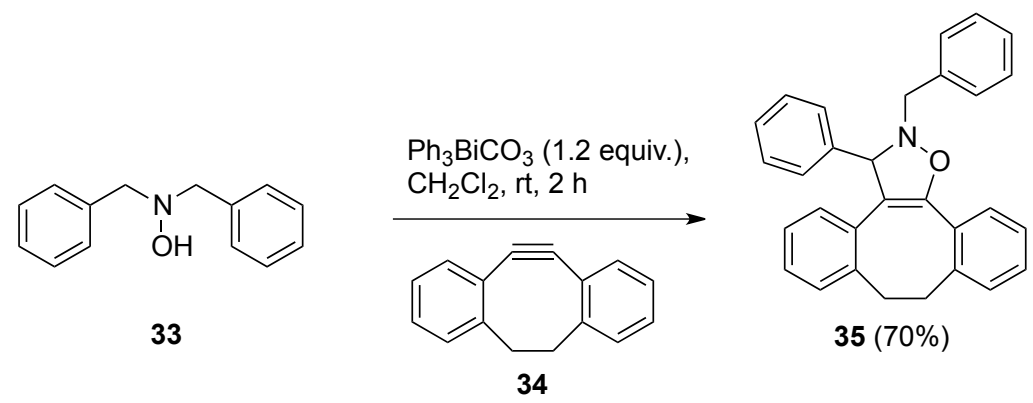

Scheme 9. One-pot oxidation/1,3-dipolar cycloaddition of 33 with $\mathrm{Ph}_{3} \mathrm{BiCO}_{3}$. of a series of $4 \mathrm{H}$-imidazole 3 -oxides $\mathbf{2 3}$ (85-90\% yield) through oxidation of hydroxylamines 22 (Scheme 6). ${ }^{25]}$

This procedure was applied in 2012 to the regioselective oxidation of the trisubstituted hydroxylamine $\mathbf{2 4}$, affording a selectivity only slightly lower with respect to the use of $\mathrm{MnO}_{2}(6: 1$ vs $8: 1)$. [26] The formation of aldonitrone $\mathbf{2 5}$ as the major product is in total agreement with the previously proposed mechanism, based on stereoelectronic effects.[15,17a] Indeed, removal of the proton in position 5 is facilitated by the anti relation with the vicinal $\mathrm{O} t \mathrm{Bu}$ group, favored with respect to the elimination of the cis-located proton in position 2 leading to ketonitrone $\mathbf{2 6}$.

The search for more sustainable oxidants prompted an investigation of the use of 2,2,6,6-tetramethyl-1-piperidinyloxy radical (TEMPO) in 2007, which promoted the selective oxidation of a highly functionalized hydroxypyrrolidine 27 (Scheme 7 ). Indeed, in this case, the kinetically favored nitrone $\mathbf{2 8}$ was obtained in a remarkable $6.7: 1$ ratio with respect to the thermodynamic nitrone $\mathbf{2 9}$ when the reaction was carried out at $0{ }^{\circ} \mathrm{C}$ for $3 \mathrm{~h}$, while the use of $\mathrm{MnO}_{2}$ at $80{ }^{\circ} \mathrm{C}$ furnished a 1:1 mixture of the two nitrones. ${ }^{[27]}$

Based on their extensive use in organic synthesis as selective oxidants and environmentally friendly reagents, ${ }^{[28]}$ we recently envisaged that hypervalent iodine compounds could be successfully applied to the oxidation of $\mathrm{N}, \mathrm{N}$-disubstituted hydroxylamines, and we reported in 2015 the reactions of a series (13 examples) of cyclic and acyclic substrates. ${ }^{[29]}$ We initially screened commercially available $o$ iodoxy benzoic acid (IBX), Dess-Martin periodinane (DMP) and diacetoxyiodobenzene (DIB), and we found IBX as the best reagent affording the desired nitrones efficiently by a simple and straightforward procedure. In particular, carbohydratederived hydroxylamines $\mathbf{3 0}$ gave nitrones 31 and 32 in excellent yields (89-100\%) and very good selectivity in favor of the aldonitrones 31 (Scheme 8).

Concerning the mechanism of this oxidation, we collected spectroscopic evidence by ${ }^{1} \mathrm{H}$ NMR studies suggesting coordination of the IBX iodine atom at nitrogen, rather than at oxygen of the hydroxylamine, in agreement with the proposal by Nicolau and co-workers for the IBX oxidation of amines. ${ }^{[30]}$ According to this mechanism, abstraction of hydrogen by IBX occurs from the less substituted carbon atom $\alpha$ to nitrogen, thus accounting for the observed high regioselectivity in favor of the aldo nitrones. ${ }^{[29]}$ We later expanded the substrate scope of IBX oxidation to differently functionalized $\mathrm{N}, \mathrm{N}$ disubstituted hydroxylamines, demonstrating wide compatibility to the presence 
of other functional groups (vinyl, allyl and alkyne). Moreover, by applying our oxidative protocol to monosubstituted hydroxylamines, the unprecedented formation of nitroso derivatives in a straightforward manner was disclosed.[31]

Due to the low toxicity of bismuth among the heavy metals, ${ }^{[32]}$ Doris and coworkers reported in 2016 about the use of triphenylbismuth carbonate for the oxidation of cyclic and acyclic (10 examples) $\mathrm{N}, \mathrm{N}$-disubstituted hydroxylamines. The reaction was carried out using 1.2 equiv. oxidant in dichloromethane at room temperature, affording excellent yields with benzyl hydroxylamines ( $>91 \%$ ), and lower yields (40-76\%) with alkyl substituted and cyclic substrates. An interesting example of one-pot oxidation/1,3-dipolar cycloaddition of hydroxylamine $\mathbf{3 3}$ to the strained benzannulated cyclooctyne $\mathbf{3 4}$ was reported, affording cycloadduct $\mathbf{3 5}$ in $\mathbf{7 0} \%$ yield after $2 \mathrm{~h}$ (Scheme 9). ${ }^{[33]}$

\section{Conclusions}

In conclusion, $N, N$-disubstituted hydroxylamines can be easily oxidized to the corresponding nitrones using a variety of stoichiometric reagents. To replace toxic $\mathrm{HgO}$ salts, which were traditionally used for this transformation, many efforts have been made in the last 25 years to develop more environmentally benign oxidants either using less toxic metal derivatives $\left(\mathrm{MnO}_{2}, \mathrm{Ph}_{3} \mathrm{BiCO}_{3}\right)$, or employing metalfree reagents $(\mathrm{NaOCl}$, TEMPO, hypervalent iodine reagents).

Due to our interest in obtaining highly hydroxylated nitrones, key building blocks for the total synthesis of natural alkaloids and their analogues, we have in particular collected examples describing the oxidation of cyclic enantiopure hydroxylamines. The high functionalization of these substrates accounts for the selectivity issue arising in the oxidation step. Indeed, when unsymmetrically substituted hydroxylamines are oxidized, two different regioselectivity problems may emerge, and this has been the object of several studies. On one hand, formation of aldo versus keto nitrones is guided by a balance of kinetic and thermodynamic effects, and in particular the use of highly hindered oxidants such as IBX allowed to obtain the aldo nitrones in very good selectivity. On the other hand, the presence of an electron withdrawing substituent in position $\mathrm{C}(3)$ of the hydroxypyrrolidine can drive the formation of one isomer over the other, with the obtainment of the major product being guided by the substrate structure quite independently on the oxidant employed.

The newly developed procedures are simple and user-friendly, require facile work-up and often allow access to pure nitrones without the need of further purification. However, efforts to replace toxic solvents such as dichloromethane are desirable and represent a challenge for the future.

\section{Acknowledgements}

We thank Fondazione Donegani/Accademia dei Lincei for a fellowship to C.M.

Received: July 27, 2017

[1] a) A. Dhainaut, A. Tizot, E. Raimbaud, B. Lockhart, P. Lestage, S. Goldstein, J. Med. Chem. 2000, 43, 2165; b) R. A. Floyd, H. K. Chandru, T. He, R. Towner, Anti-Cancer Agents Med. Chem. 2011, 11, 373; c) R. A. Floyd, H. C. Castro Faria Neto, G. A. Zimmerman, K. Hensley, R. A. Towner, Free Radical Biol. Med. 2013, 62, 145.

[2] Reviews: a) P. Merino, Sci. Synth. 2004, 27, 511; b) P. Merino, S. Franco, F. L. Merchan, T. Tejero, Synlett 2000, 442; c) M. Lombardo, C. Trombini, Synthesis 2000, 759.

[3] a) J. J. Tufariello, in '1,3-Dipolar Cycloaddition Chemistry', vol. 2, Ed. A. Padwa, John Wiley \& Sons, New York, 1984, p. 83; b) K. V. Gothelf, K. A. Jørgensen, Chem. Rev. 1998, 98, 863; c) A. Brandi, F. Cardona, S. Cicchi, F. M. Cordero, A. Goti, Chem. Eur. J. 2009, 15, 7808 .

[4] a) G. Masson, S. Py, Y. Vallée, Angew. Chem. Int. Ed. 2002, 41, 1772; b) I. S. Young, M. A. Kerr, Angew. Chem. Int. Ed. 2003, 42, 3023.

[5] Reviews: a) F. Cardona, A. Goti, Chem. Int. Ed. 2005, 44, 7832; b) J. Yang, Synlett 2012, 2293; c) L. L. Anderson, Asian J. Org. Chem. 2016, 5, 9.

[6] a) H. Mitsui, S. Zenki, T. Shiota, S.-I. Murahashi, J. Chem. Soc. Chem. Commun. 1984, 874; b) S.-I. Murahashi, H. Mitsui, T. Shiota, T. Tsuda, S. Watanabe, J. Org. Chem. 1990, 55, 1736; c) S.-I. Murahashi, T. Shiota, Y. Imada, Org. Synth. 1992, 70, 265; d) S.-I. Murahashi, T. Shiota, Tetrahedron Lett. 1987, 28, 2383; e) E. Marcantoni, M. Petrini, O. Polimanti, Tetrahedron Lett. 1995, 36, 3561; f) A. Goti, L. Nannelli, Tetrahedron Lett. 1996, 37, 6025; g) A. Goti, F. Cardona, G. Soldaini, Org. Synth. 2005, 81, 204; h) R. W. Murray, K. Iyanar, J. Chen, J. T. Wearing, J. Org. Chem. 1996, 61, 8099; i) S. Yamazaki, Bull. Chem. Soc., Jpn. 1997, 70, 877; j) M. Forcato, W. A. Nugent, G. Licini, Tetrahedron Lett. 2003, 44, 49.

[7] a) E. Breuer in 'The Chemistry of Amino, Nitroso and Nitro Compounds and Their Derivatives', Ed. S. Patai, Wiley: New York, 1982, p. 459; b) J. A. Grigoriev in 'Nitrile Oxides, Nitrones, and Nitronates in Organic Synthesis', Ed. H. Feuer, John Wiley and Sons, New Jersey, 2008, p. 129.

[8] a) G. Soldaini, F. Cardona, A. Goti, Org. Lett. 2007, 9, 473; b) F. Cardona, M. Bonanni. G. Soldaini, A. Goti, ChemSusChem 2008, 1, 327.

[9] S. Katahara, S. Kobayashi, K. Fujita, T. Matsumoto, T. Sato, N. Chida, J. Am. Chem. Soc. 2016, 138, 5246.

[10] 'Oxidation of alcohols to aldehydes and ketones', Eds G. Tojo, M. Fernández, Springer, New York, 2010.

[11] For a recent review on aerobic oxidation of alcohols see: C. Parmeggiani, C. Matassini, F. Cardona, Green Chem. 2017, 19, 2030 and references cited therein.

[12] a) J. Hamer, A. Macaluso, Chem. Rev. 1964, 64, 473 ; b) P. N. Confalone, Huie, E. M. Organic Reactions 1988, 36, 1.
[13] a) R. Bonnett, R. F. C. Brown, V. M. Clark; I. O Sutherland, A. Todd, J. Chem. Soc. 1959, 2094; b) R. F. C. Brown, V. M. Clark, I. O. Sutherland, A. Todd, J. Chem. Soc. 1959, 2109; c) P. A S. Smith, S. E. Gloyer, J. Org. Chem. 1975, 40, 2508; d) E. C. Taylor, F. Yoneda, Chem. Commun. (London) 1967, 199.

[14] S. Cicchi, I. Höld, A. Brandi, J. Org. Chem. 1993, 58, 5274.

[15] S. Cicchi, A. Goti, A. Brandi, J. Org. Chem. 1995, 60, 4743.

[16] J. Revuelta, S. Cicchi, A. Goti, A. Brandi, Synthesis 2007, 485.

[17] a) A. Goti, S. Cicchi, V. Fedi, L. Nannelli, A. Brandi, J. Org. Chem. 1997, 62, 3119; b) A. Goti, V. Fedi, L. Nannelli, F. De Sarlo, A. Brandi, Synlett 1997, 577.

[18] a) A. Goti, M. Cacciarini, F: Cardona, A Brandi, Tetrahedron Lett. 1999, 40, 2853; b) A. Goti, S. Cicchi, M. Cacciarini, F. Cardona, V. Fedi, A. Brandi, Eur J. Org. Chem. 2000, 3633.

[19] S. Cicchi, M. Corsi, A. Goti, J. Org. Chem. 1999, 64, 7243.

[20] S. Cicchi, M. Marradi, A. Goti, A. Brandi, Tetrahedron Lett. 2001, 42, 6503.

[21] S. A. Ali, S. M. Azhar Hashmi, M. N. Siddiqui, M. I. M. Waazer, Tetrahedron 1996, 52, 14917.

[22] S. A. Ali, M. I. M. Waazer, Tetrahedron 1993, 49, 4339 .

[23] a) S. Cicchi, M. Corsi, M. Marradi, A. Goti, Tetrahedron Lett. 2002, 43, 2741; b) S. Cicchi, M. Marradi, C. Corsi, C. Faggi, A. Goti, Eur. J. Org. Chem. 2003, 4152.

[24] S. A. Ali, S. M. A. Hashmi, M. I. M. Wazeer, Tetrahedron Lett. 1998, 39, 1255.

[25] a) I. A. Grigor'ev, I. A. Kirilyuk, L. B. Volodarskii, Chem. Heterocycl. Compd. 1988 24, 1355. b) I. A. Kirilyuk, T. G. Shevelev, D. A. Morozov, E. L. Khromovskih, N. G. Skuridin, V. V. Khramtsov, I. A. Grigor'ev, Synthesis 2003, 871 .

[26] D. A. Morozov, I. A. Kirilyuk, D. A. Komarov, A. Goti, I. Yu. Bagryanska, N. V. Kuratieva, I. A. Grigor'ev, J. Org. Chem. 2012, 77, 10688.

[27] P. Merino, I. Delso, T. Tejero, F. Cardona, A. Goti, Synlett 2007, 2651.

[28] a) D. B. Dess, J. C. Martin, J. Am. Chem. Soc. 1991, 113, 7277; b) A. Duschek, F. Kirsch, Angew. Chem., Int. Ed. 2011, 50, 1524; c) A. Yoshimura, V. V. Zhdankin, Chem. Rev. 2016, 116, 3328.

[29] C. Matassini, C. Parmeggiani, F. Cardona, A Goti, Org. Lett. 2015, 17, 4082.

[30] a) K. C. Nicolau, C. J. N. Mathison, T. Montagnon, Angew. Chem., Int. Ed. 2003, 42, 4077; b) K. C. Nicolau, C. J. N. Mathison, T. Montagnon, J. Am. Chem. Soc. 2004, 126, 5192.

[31] C. Parmeggiani, C. Matassini, F. Cardona, A. Goti, Synthesis 2017, 49, 2890.

[32] R. Mohan, Nat. Chem. 2010, 2, 336.

[33] D.-V. Nguyen, P. Prakash, E. Gravel, E. Doris, RSC Adv. 2016, 6, 89238. 